\title{
Théologiques
}

\section{Le « spirituel » et le " religieux » à l'épreuve de la}

\section{transcendance}

\section{Robert Jacques}

Volume 7, numéro 1, printemps 1999

Les trente ans de la bioéthique

URI : https://id.erudit.org/iderudit/024975ar

DOI : https://doi.org/10.7202/024975ar

Aller au sommaire du numéro

Éditeur(s)

Faculté de théologie de l'Université de Montréal

ISSN

1188-7109 (imprimé)

1492-1413 (numérique)

Découvrir la revue

Citer cet article

Jacques, R. (1999). Le " spirituel » et le " religieux » à l'épreuve de la

transcendance. Théologiques, 7(1), 89-106. https://doi.org/10.7202/024975ar d'utilisation que vous pouvez consulter en ligne.

https://apropos.erudit.org/fr/usagers/politique-dutilisation/ 


\title{
Le "spirituel " et le " religieux" à l'épreuve de la transcendance
}

\author{
Robert JACQUES \\ Faculté de théologie \\ Université de Montréal
}

«Gardez-vous du levain des Pharisiens et d'Hérode. »

Marc 8,15

Une jeune femme, coopérante en Bolivie, m'écrivait récemment au sujet de son mariage : "La cérémonie eut lieu dans le jardin d'un collège (on n'avait pas l'envie de s'enfermer dans une église) et fut très vivante grâce au prêtre [...], quelqu'un d'ouvert et de spirituel (mais non de "religieux", si vous voyez la différence?!). " Une photographie des nouveaux mariés accompagnait la lettre. On y voyait la jeune épouse émergeant à peine d'une robe blanche à crinolines et multiples dentelles des plus traditionnelles.

Ses mots traduisent bien la perception spontanée dans les socié tés occidentales actuelles de la religion et de la spiritualité. Ces dernières, dans les lignes écrites par cette jeune femme, diffèrent en fait tellement qu'il est probablement plus juste de dire qu'elles s'opposent. Le religieux et l'église qui est son lieu de manifestation enferment, emprisonnent. Le spirituel au contraire est ouverture qu'une cérémonie dans un jardin exprime, semble-t-il, beaucoup mieux. Faut-il y voir une nostalgie inconsciente de l'oasis mythique où vivait le premier couple biblique? Un désir de retrouver un instant leur innocence supposée? Faut-il comprendre le spirituel comme ce qui était avant le «péché » et celui-ci alors comme l'instigateur de la religion étouffante? Il est un peu cruel de ma part d'en faire dire tant à ces quelques remarques d'une jeune mariée radieuse de partager son bonheur. Malgré leur brièveté, elles en disent long... 


\section{Spiritualité et religion : un long conflit}

La critique de la religion asphyxiante n'est pas nouvelle. Déjà, des prophètes du Premier Testament s'en sont fait les témoins par leurs reproches à ceux et celles qui n'honoraient Dieu que des lèvres. Jésus reprit ces critiques à l'égard des Pharisiens et leurs scribes dont les traditions annulaient la parole de Dieu (voir Mc 7,13). Cette critique rebondit encore sous les lignes de Paul opposant Loi et Évangile, la chair et l'esprit. Une histoire du christianisme pourrait être écrite, avec pour intrigue, l'antagonisme entre le religieux et le spirituel. Les réformes ecclésiales successives qui ont jalonné les deux mille ans de son existence en témoignent largement. Ces réformes étaient des autocritiques dont les initiateurs ne manquaient pas d'en attribuer la responsabilité à l'Esprit-Saint lui-même.

Plus récemment, la modernité laïque a aussi formulé sa critique de la religion. Elle n'était plus l'œuvre d'un Esprit de sainteté et de renouvellement, mais l'œuvre de la raison autonome, de la conscience révolutionnaire, d'une volonté de libération pour les peuples et pour les individus. La religion - c'est-à-dire, dans la ligne de mire des maîtres du soupçon, la religion chrétienne - était tantôt "opium " pour le peuple, tantôt " platonisme " pour le même peuple, ou encore "néurose obsessionnelle». Des théologiens, surtout de tradition protestante, ont entendu ces contestations radicales. Ainsi Karl Barth formula, à la manière de Paul et de Luther, une opposition entre foi et religion, interprétant cette dernière comme entreprise humaine d'autojustification. Bonhoeffer, dans ses ultimes lettres de prison, esquissa les contours d'une foi, d'un christianisme non religieux.

Mais la critique de la religion par la jeune mariée ne se réclame pas de la modernité sonnant le glas de la pensée judéo-chrétienne et encore moins d'une apologétique du christianisme. Elle renvoie dos à dos ce que pendant des siècles de foi chrétienne on a tenu pour des quasi synonymes, malgré l'incessante tension entre ces termes et leur interprétation. La religion était le lieu de la spiritualité. Nul n'aurait imaginé que le spirituel pourrait se vivre hors du religieux. Le catholicisme, pour ne s'en tenir qu'à celui-ci, proposait plusieurs "spiritualités " identifiées à de grandes figures de l'expérience croyante : François d'Assise, Dominique, Thérèse d'Avila, Jean de la 
Croix, Ignace de Loyola, etc. Mais le religieux absorbait le spirituel, en contrôlait les excès mystiques, contrôle que d'aucuns soupçonnaient être la victoire de la lettre sur l'Esprit (voir 2 Co 3,6).

Aujourd'hui, la spiritualité secoue le joug du religieux; elle s'en affranchit au nom d'un affrontement entre ouverture et enfermement, comme en témoignent les lignes de cette jeune femme. En témoigne également l'essai quasi-pamphlétaire L'Homme-Dieu ou le sens de la vie de Luc Ferry. Celui-ci appelle « à une spiritualité authentique, débarrassée de ses oripeaux théologiques, enracinée dans l'homme et non dans une représentation dogmatique de la divinité ${ }^{1}$ ". Sous la plume de Ferry, le fossé entre spiritualité et religion est creusé par l'opposition entre l'exigence d'authenticité - synonyme ici d'intériorité de la liberté et de l'autonomie modernes et l'extériorité des autorités et des dogmatismes habillés d'oripeaux - donc synonyme d'inauthenticité2.

Spiritualité et religion qui, encore récemment, formaient un couple souvent malheureux, sont désormais aux yeux de plusieurs des "sœurs ennemies" engendrant des dualismes irréductibles : ouverture/fermeture, authenticitélinauthenticité qui ne laissent plus une grande

1 L. FERRY, L'Homme-Dieu ou le sens de la vie, Paris, Grasset (Livre de Poche, 14261), 1997, p. 34. Parlant d'une paradoxale "transcendance dans l'immanence » (p.38), cet auteur veut "répondre aux exigences d'une pensée moderne de la transcendance : cette dernière est donnée, hors de tout argument d'autorité, dans l'immanence au vécu subjectif et à partir de lui." (p. 40). L'argument d'autorité y est sans cesse rejeté parce qu'il ressortit à l'extériorité : "il s'agit encore de décider si les faits de la religion doivent conserver ou non leur statut d'extériorité radicale à l'égard des êtres humains ou, à l'inverse, être débarrassés de leurs oripeaux extérieurs en vue d'une intériorisation de leur signification authentique " (p. 58). Une pensée libre, sans extériorité, est-elle possible? La médiation du langage, sans être coercitive, est déjà chemin balisé de l'argumentation; et la liberté est réponse motivée par des désirs, des besoins, des mobiles. On remarquera au passage que l'opposition entre spiritualité et religion rappelle celle établie actuellement entre éthique et morale.

2 Le Petit Robert écrit au sujet du terme oripeau: "ornement de faux or ou de faux argent ", "faux éclat, apparence brillante et trompeuse ", "vieux habits dont un reste de clinquant fait ressortir l'usure. " 
liberté de choix! La revendication d'une spiritualité libérée du religieux en vient à ressembler à une entreprise de purification, puisqu'elle appelle à se " débarrasser » de ce qui est jugé fausseté ou trompeuse apparence. Attesterait-elle à son insu d'une attitude pharisienne ou cathare? De la tentation obsédante de la pureté, tentation génératrice de tant de mythes anciens ou contemporains ${ }^{3}$ ? Longtemps, trop longtemps, le religieux s'est cru le « maitre et possesseur » de la spiritualité; aujourd'hui, celle-ci prétend régner sur tout ce qui évoque quelque transcendance 4 . Comment entendre la distinction entre le spirituel et le religieux - distinction bien réelle puisque le vocabulaire lui-même la manifeste - sans tomber dans des antagonismes insurmontables? Comment rendre féconde leur différence?

Conscient de pécher contre la rectitude politique qui, en bien des lieux, remplace désormais la moralité judéo-chrétienne, je me refuse à endosser sans aucune interrogation la « différence " affirmée cavalièrement entre le religieux et le spirituel; il faut s'y refuser parce que la différence postulée est synonyme de dénégation ou de mépris de l'un à l'égard de l'autre. Certes distincts, différents, le religieux et le spirituel peuvent-ils engager d'autres relations que celles de la méfiance et de l'exclusion? Comment faire entendre leur «différence» véritable, comment faire entendre ce que l'un et l'autre portent d'appel à l'authenticité? L'étymologie l'enseigne : la

3 Tout récit fondateur d'une communauté postule à son origine ou à son telos un idéal de pureté : ainsi peut-on comprendre le texte de Genèse 2-3 où le premier couple expérimente une innocence avant la chute ou les projets de l'homme nouveau purifié de la tare bourgeoise de certaines idéologies de ce siècle.

4 Voir D.N. ELKINS et al., "Toward a Humanistic-Phenomenological Spirituality. Definition, Description, and Measurement », Journal of Humanistic Psychology 28/4 (1988) 5-18; p. 12 : "Our humanistic model would assess spirituality more comprehensively and would not equate it with narrow religious beliefs, rituals, and practices.". [Notre modèle humaniste de la spiritualité voudrait rendre compte du caractère englobant de celle-ci et de sa soustraction aux croyances, pratiques et rituels religieux étroits.] 
"férence " disait anciennement "porter dans son ventre "! Parler de différence, c'est suggérer des manières variées de «porter du sens ».

\section{L'authenticité en tant que quête du sens de l'existence}

Les hommes et les femmes de la (post-)modernité sont mêlés à une "sombre affaire» de sens. La philosophie existentialiste a déplacé la question du sens. Longtemps, poser la question du sens était s'interroger sur le vouloir-dire, le propos, l'intention des textes, des œuvres. L'herméneutique était la discipline guidant cette inter rogation. Du sens, il y en avait dans les œuvres du passé. Il était demandé aux humains de les déchiffrer afin d'y découvrir ainsi le sens de leur propre existence. De cette quête de sens, l'évangile de Luc en témoigne: les deux compagnons en route vers Emmaüs accomplissent ce travail herméneutique des Écritures concernant celui dont il portait le deuil (voir 24,27).

Avec Martin Heidegger, l'existence elle-même est auto-interprétation. L'être humain est cet être sans cesse convoqué à la compréhension de soi. Cette compréhension de soi est sa responsabilité pour une vie authentique. Sa propre existence est le texte à lire, à interpréter ${ }^{5}$. Le sens y réside désormais. Le déplacement n'est pas insignifiant. L'herméneutique de soi autorise, exige même la remise en question de toute autorité : ce qui jusqu'à présent prétendait donner, être auteur ${ }^{6}$ de sens est soupçonné de n'être que bavardages du " on" et de menacer ainsi l'être humain d'inauthenticité. Ce déplacement - ce qui est à comprendre n'est plus au-delà de soi, mais soi tout simplement - définit une nouvelle configuration de la spiritualité en laquelle celle-ci s'exile de plus en plus du religieux. La diffé-

5 Voir P. RICCEUR, "Contribution d'une réflexion sur le langage à une théologie de la parole", dans R. BARTHES et al., Exégèse et herméneutique. Paris, Seuil, 1971, pp. 301-319; aux pp. 315s : «le langage serait vain s'il n'avait des racines ontologiques dans la structure même de l'être. C'est là, me semble-t-il, l'apport fondamental de la philosophie de Heidegger [...] du comprendre, en quelque sorte prélinguistique, on s'élève au discours proprement dit, à l'énonciation, en passant par le mode clé de l'interprétation, lequel appartient à l'existence comme telle. »

6 Du latin auctoritas, caractère de l'auctor (d'où le français, auteur). 
renciation - jusqu'à la confrontation ou l'éclatement - du spirituel et du religieux est ainsi la résultante d'une quête de sens, synonyme de l'existence même. Le sous-titre de l'essai de Luc Ferry l'annonce clairement : "le sens de la vie "; un mariage célébré dans un jardin témoigne également d'une recherche de signification.

Que la quête actuelle de sens inscrive une ligne de partage, de fracture même, entre le religieux et le spirituel constitue un fait nouveau et majeur. Si la religion est déclarée enfermement ou inauthenticité drapée dans ses " oripeaux» - des haillons, diraient les plus cyniques - , c'est qu'elle apparaît désormais impuissante à soutenir les interrogations des humains sur le sens de leur existence dans un monde «désenchanté ». D'ailleurs, la religion n'est pas seule à vivre cette impuissance. Les grandes idéologies et utopies - celles-là même qui ont cru se substituer à « l'illusion religieuse »-partagent désormais sa stérilité. Le sens qu'elles promettaient n'interpelle plus, n'interrompt plus le va-et-vient des humains. Ce sens et l'espoir qu'il suscitait enchantaient le monde; celui-ci avait encore un avenir. Qui oserait aujourd'hui prophétiser des temps nouveaux? Si du sens est encore possible, il est du ressort de la spiritualité dépouillée de quelque «structure religieuse ${ }^{7}$ ».

L'intérêt non religieux pour la spiritualité - pour la question du sens - est partagé désormais par beaucoup. Ainsi des chercheurs des Hautes études commerciales réfléchissent sur La Quête du sens, sur le désir d'authenticité des individus dans leur vie personnelle et au sein des organisations ${ }^{8}$. La signification même de cet intérêt qui revêt des formes toujours plus nombreuses et étonnantes n'est pas évidente; elle suscite une abondante production littéraire ${ }^{9}$. Cet intérêt manifeste

L. FERRY, L'Homme-Dieu ou le sens de la vie, écrit à la page 18 : "les diverses variantes du communisme ne pouvaient offrir de sens qu'en vertu d'une authentique structure religieuse aujourd'hui révolue: elles impliquaient, même dans leurs versions matérialistes les mieux sécularisées, l'idée d'un "Au-Delà " de la vie présente. "

8 Voir T. PAUCHANT et collaborateurs, La Quête du sens. Gérer nos organisations pour la santé des personnes, de nos sociétés et de la nature. Montréal, Québec/Amérique [Presses HEC], 1996, 359 p.

9 Voir «Le temps des religions sans Dieu », Esprit 233 (1997). 
le difficile deuil de la mort de Dieu (ou de l'idole) dans les sociétés sécularisées. Le projet d'une "divinisation de l'humain ", grâce à une « humanisation du divin», proposé par Luc Ferry dans L'HommeDieu, en est une attestation explicite. Il y a une perte à laquelle les hommes et les femmes ne peuvent se résigner.

Les voix s'exprimant aujourd'hui sur la spiritualité sont toujours plus nombreuses. Il y a tout particulièrement la voix de ceux et celles qui, dans des milieux divers, accompagnent et soignent les hommes et les femmes dont l'existence est déchirée par les souffrances de la chair ou du psychisme. Temps concret d'expérience du «bien » et du «mal », la maladie est ainsi lieu de quête de sens. L'attention à la dimension spirituelle et à la dimension religieuse des personnes soignées, si elle n'est pas à vrai dire nouvelle, n'est toutefois explicitement abordée que depuis quelques décennies. Divers facteurs ont contribué à ce souci : la déception devant les limites des sciences médicales traditionnelles, la critique vive du dualisme cartésien, l'avènement d'une pensée dite holistique, des thérapies parallèles, etc. Aussi dans la prolifération actuelle de recherches sur la spiritualité et la religion, les études en sciences infirmières, comme nous le verrons au paragraphe suivant, sont un témoin éloquent de la tension entre celles-là.

\section{Des besoins spirituels et des besoins religieux}

La réflexion sur la dimension spirituelle et la dimension religieuse dans les soins de santé doit beaucoup au modèle d'interven tion infirmière proposée par Virginia Henderson dans les années soixante-dix. Dans ce modèle identifiant quatorze besoins fondamentaux de la personne malade, V. Henderson nommait expressément celui de "pratiquer sa religion ou d'agir selon sa conception du bien et du mal ». Une première distinction était ainsi proposée entre la pratique religieuse et l'action morale. Cette formulation, et d'autres également, a donné lieu à de nombreuses recherches ${ }^{10}$. Au fil de celles-ci, la distinction entre religion et

10 Pour un aperçu de la littérature américaine consacrée à la question, voir B. KOZIER et al., Fundamentals of Nursing. Concepts, Process, and Practice. Redwood City, Addison-Wesley Nursing, 1995, pp. 323-324. 
spiritualité est devenue l'objet d'un travail intense de définition au service de l'intervention infirmière, l'objectif essentiel étant de proposer des concepts et des critères opérationnels précis pour l'établissement des besoins, la prestation de soins et l'évaluation de l'action accomplie. Cette finalité pratique doit nous rappeler que la rigueur lexicale poursuivie n'est pas celle du philosophe ou du théologien. Elle est celle d'acteurs et d'actrices dans un milieu professionnel donné, animés d'une sollicitude à l'égard de personnes impliquées bien souvent dans des luttes pour leur survie. Mais ce milieu professionnel participe à la culture ambiante. Aussi l'écoute attentive des définitions de la spiritualité et de la religion qui s'y dessinent peut mettre à jour des défis pastoraux et des questionnements théologiques provocateurs ${ }^{11}$.

Des articles récents ont recueilli dans la littérature infirmière américaine divers usages des termes en question. En voici un échantillonnage significatif. Les premiers emplois cités sont tirés d'un manuel d'enseignement de soins infirmiers.

La spiritualité est multidimensionnelle; les deux dimensions les plus fréquemment citées sont la dimension existentielle et la dimension religieuse. La dimension existentielle vise le but (purpose) et la signification (meaning) de l'existence; la dimension religieuse concerne la relation personnelle (one's relationship) à Dieu ou à une Puissance supérieure ${ }^{12}$.

11 Si l'on définit le souci pastoral, comme étant celui de l'incarnation de l'Évangile du salut donné en Jésus dans le terreau réel de l'existence, ce souci exige une méditation profonde et critique de l'attente de libération formulée dans les compréhensions de soi que comportent les définitions que les hommes et les femmes emploient pour (se) dire; les expressions dimension spirituelle et dimension religieuse sont de celles-là. Dit autrement, les définitions culturelles proposées sont-elles réductrices ou émancipatrices des humains dont elles veulent dire quelque chose?

12 J.R. MICKLEY et al., "Spiritual well-being, religiousness, and hope among women with breast cancer ", Image : Journal of Nursing Scholarship, Winter 1992, 24, p. 267; cité dans B. KOZIER et al. Fundamentals of Nursing, op.cit., p. 312. 
Certains besoins spirituels sous-tendent (underlies) toutes les religions : (a) le besoin de sens et de but, (b) le besoin d'amour et de relation (relatedness), et le besoin de pardonner ${ }^{13}$.

La religion est un système organisé de culte (worship). Les religions ont des croyances centrales, des rituels et des pratiques habituellement reliés à la mort, au mariage et au salut. Elles comportent aussi souvent des règles de conduite applicable à la vie quotidienne. Plusieurs personnes satisfont leurs besoins spiri tuels à travers une religion spécifique ou un cadre (framework) religieux ${ }^{14}$.

Ces extraits invitent déjà à quelques remarques. Ils nous présentent une interprétation ample de la spiritualité : celle-ci inclut, englobe l'expérience religieuse. Parce qu'elle est affaire de sens et de but, de relation et d'affectivité, son périmètre est indéfini; ce qui lui confère un caractère illimité et équivoque. La religion, au contraire, est rigoureusement déterminée; ses contours sont précis, son contenu est composé d'éléments factuels faciles à repérer: croyances, rites, pratiques, règles ordonnés dans un cadre ou système. On peut entendre ici en écho le couple ouverture - enfermement suggéré par les lignes écrites par ma jeune correspondante.

Julia D. Emblen, dans « Religion and Spirituality Defined According to Current Use in Nursing Literature ${ }^{15}$ ", retrace chronologiquement les acceptions diverses de ces deux mots tirées de publications s'échelonnant de 1963 à 1989. Cette présentation diachronique est révélatrice des déplacements dans la compréhension culturelle des réalités visées. Voici quelques définitions répertoriées:

13 J.A. SHELLEY and S. FISH, Spiritual Care: The Nurse's Role. $3 \mathrm{~d}$ ed. Downers Grove, InterVarsity Press, 1988, pp. 40-53, cité dans B. KOZIER et al. Fundamentals of Nursing, op.cit., p. 312.

14 B. KOZIER et al. Fundamentals of Nursing, op.cit., p. 312.

15 Joumal of Professional Nursing 8/1 (1992) 41-47. 


\section{Spiritualité :}

"la qualité de ces forces qui nous animent, ou les principes essentiels qui nous influencent. Le spirituel n'implique pas nécessairement le religieux. Le spirituel s'oppose au biologique et à la mécanique. " (1970; p. 43)

" le fait d'avoir une relation dynamique et personnelle avec Dieu. " (1980; p. 43)

«principe vital qui habite l'être entier de la personne en toutes ses dimensions: volontaire, émotionnelle, morale et éthique, intellectuelle et aussi physique, et l'ouvre à des valeurs transcendantes. " (1981; p. 43)

"ce qui inspire le désir de transcender le domaine matériel." (1982; p. 43)

" une qualité qui surpasse l'affiliation religieuse et suscite l'inspiration, la révérence, l'étonnement (awe), la signification et le but, même lorsque la personne ne croit pas en Dieu. La dimension spirituelle est la quête d'harmonie avec l'univers et comporte des questions au sujet de l'infini. » (1985; p. 43)

«principe vital qui habite et anime tout l'être de la personne et qui intègre et transcende sa nature biologique et psychosociale. " (1987; p. 45)

\section{Religion:}

"la reconnaissance d'une dépendance ultime de l'être humain à l'égard d'un être supérieur. » (1963; p. 42)

"système organisé de culte (worship) caractérisé par des croyances que la personne professe; des normes morales réglant la conduite des membres d'un système religieux, des rites et des pratiques en usage dans le système de culte. » (1980; p. 42)

" croyance en une force surnaturelle ou divine ayant puissance sur l'univers et exigeant culte et obéissance; un système de croyances; un code ou une philosophie éthique; un ensemble de 
pratiques accomplies; une affiliation religieuse; la quête consciente de tout objet que la personne tient pour suprême. » (1985; p. 42)

« système (body) de pensée et d'expérience concernant les problèmes fondamentaux de l'existence; c'est un système organisé de croyance (faith). » (1987; p. 42)

Les remarques proposées précédemment s'appliquent à ce nouvel ensemble de citations. Il n'est pas besoin de les répéter. La présentation chronologique souligne le fossé se creusant entre religion et spiritualité; alors que celle-ci pouvait désigner encore en 1980 «une relation personnelle et dynamique avec Dieu ", elle est, dans les années subséquentes, toujours plus comprise en tant que vitalité anonyme de surpassement. De même, le religieux est délesté de son caractère relationnel pour "s'incarner ${ }^{16}$ ", se chosifier dans des croyances, des normes, des pratiques, des rites, etc. Comme le note J. Emblen, les définitions de la religion forment une constellation de six mots clés : système, croyances, organisé, personne, culte, pratiques -, alors que la spiritualité est dessinée par les mots: personnel, vie, principe, animation, être, Dieu (dieu), qualité, relation et transcendant ${ }^{17}$.

L'emploi fréquent de ce dernier terme doit retenir l'attention. Surtout utilisé en tant que verbe dans ces quelques extraits, il importe de cerner les bornes de sa signification effective. Il est apparenté ici à d'autres verbes: surpasser, s'opposer. En tant que verbe, il désigne l'action d'un sujet à l'égard de et à l'encontre de réalités de son existence: le matériel, le biologique, le mécanique, le psychosocial, l'affiliation religieuse. Action de dépassement, le «transcender " est contestation, transgression de ces réalités perçues et vécues par le sujet en tant que limites ${ }^{18}$. Étonnamment à un moment où la pensée

16 Alors que la première définition suggérait, dans un langage proche de F. Schleiermacher, une "dépendance ultime de l'être humain à l'égard d'un être supérieur ", la dernière parle d'un corps (body) organisé!

17 Voir J. EMBLEN, op.cit., p. 41.

18 Transcender et transgresser sont ici des quasi synonymes: outre le préfixe trans qui leur est commun, la racine latine de l'un et l'autre suggère l'idée de montée (scendere), de gradation (-gres, de gradus). Le pré- 
holistique et l'harmonie unificatrice sont promues par de nombreux discours culturels dont fait partie la réflexion actuelle sur la spiritualité, cette dernière réintroduit un dualisme confrontant spirituel et matériel. On ne peut s'empêcher de penser à celui de l'âme et du corps tant reproché à Descartes.

Ces remarques soulèvent dès lors quelques soupçons quant à la compréhension présente de la spiritualité. N'y a-t-il pas une certaine contradiction à instaurer une opposition entre spirituel et matériel, biologique, etc. et à tenir par ailleurs que les valeurs spirituelles ont une signification naturelle? ${ }^{19}$ La nature n'évoque-t-elle pas en premier lieu le biologique, le matériel? Dans sa volonté de secouer le joug du religieux, le questionnement sur la spiritualité répète à son insu des thématiques autrefois développées par la théologie chrétienne traditionnelle : le dualisme du corps et de l'âme, un mépris (inconscient?) de la matière, la capacité "naturelle " du divin (capax dei), la quête fusionnelle avec la totalité, une certaine abnégation de soi dans l'aspiration à un Soi englobant ${ }^{20}$, etc. Ces questions quelque peu impertinentes indiquent que le travail de clarifi-

fixe trans place le verbe transcender dans l'orbite de la pensée contemporaine valorisant le transpersonnel, le transculturel.

19 A. MASLOW, qui a beaucoup contribué à l'intérêt récent pour la spiritualité, écrivait dans Religious, Values and Peak Experiences. New York, Viking, 1970, p. 33: «I want to demonstrate that spiritual values have naturalistic meaning, that they are not the exclusive possession of organized churches, that they do not need supernatural concepts to validate them, that they are well within the jurisdiction of a suitably enlarged science, and that, therefore they are the general responsability of all mankind. " On croirait lire en ces lignes le manifeste qui a inauguré l'effort de distinction rigoureuse entre spiritualité et religion.

20 Voir D.N. ELKINS et al, "Toward a Humanistic-Phenomenological Spirituality. Definition, Description and Measurement», Journal of Humanistic Psychology 28/4 (1988), p. 10: "The spiritual person has an experientially based belief that there is a transcendent dimension to life. The actuel content of this belief may range from the traditional view of a personal God to a psychological view that the "transcendent dimension " is simply a natural extension of the conscious self into the regions of the unsconscious or Greater Self." 
cation des notions reste encore à faire; les définitions actuelles de la spiritualité suggèrent une réhabilitation du religieux dont elles veulent prendre distance : la frontière qui les sépare n'est pas aussi nette que les définitions données ci-dessus semblent le prétendre.

Autre interrogation impertinente: la spiritualité est recherche d'unification, comme l'atteste le désir « d'harmonie avec l'univers "; estce ici se profilant sous de nouvelles guises l'antique aspiration mystique à l'Un? Quête de l'extase, de la sortie de soi dans des " expériences-sommets " à la crête desquelles le sujet se retrouve seul en fuite "vers le Seul", émigration "vers l'Un dont [ill a découvert en soi une étincelle 21 ". La transcendance désirée comporte ainsi le risque d'une occultation de l'altérité concrète de l'autre: quelle place pour celui-ci dans une quête de soi qui prend la voie de l'exploration de sa participation à l'Un, dans l'ignorance du difficile mais incontournable chemin de la rencontre de l'autre? L'accomplissement ou la réalisation de soi - nouveau mot d'ordre ou nouvelle expression du salut - n'est plus tenu au détour de l'écoute de la parole de l'autre. Le sujet, par son usage métissant les diverses traditions spirituelles et religieuses, demande à celles-ci des échos à ses expériences intimes, à son monde intérieur; elles ne sont pas écoutées dans leurs possibilités de "déployer un monde nouveau, des possibilités nouvelles d'existence » devant lui, déploiement qui exige une distanciation à soi et une appropriation de ces possibilités 22 . Le terme appropriation indique bien qu'il est question de faire siennes des possibilités reconnues comme n'étant pas propres à soi. L'expérience spirituelle authentique serait celle qui maintient l'être humain dans

21 J.-P. JossuA, Le Dieu de la foi chrétienne. Paris, Cerf; Montréal, Fides, 1989, p. 40.

22 On aura reconnu ici quelques éléments essentiels de la réflexion herméneutique de Paul Ricœur; voir $D u$ texte à l'action. Essais d'herméneutique, II. Paris, Seuil, 1986, pp. 101-133. La demande spirituelle actuelle est demande de sens, demande de se comprendre: les travaux herméneutiques de Gadamer et de Ricœur soulignent abondamment que la compréhension de soi est liée au détour de l'écoute de l'autre opérant non le dépassement, mais la distanciation à soi; Gadamer voit même en celleci la dimension spirituelle de l'être humain, voir Philosophie de la santé. Paris, Grasset-Mollat, 1998, pp. 60-67. 
cet écart à soi où il lui est donné de résister à tous les discours prétendant détenir le sens ultime de son existence. L'actuel retour du spirituel indique bien la difficile distance à soi : il est demandé aux croyances de tenir lieu de "ciment " identitaire des personnes et des collectivités. La métamorphose des croyances en identités est tout aussi funeste que la chosification qui accompagne les systèmes religieux.

Dernière question: la transcendance visée dans les définitions de la spiritualité est surpassement; cette synonymie est confirmée par l'étymologie où transcendere signifie «passer par-dessus ». Ce thème du surpassement est présent chez des théologiens chrétiens. Ainsi Micheline Laguë dans un article récent cite Sébatian Kappen pour qui la spiritualité peut se définir comme "la manière dont les humains se dépassent pour atteindre les possibilités ultimes de leur existence » et Adolphe Gesché qui affirme que "sauver, c'est amener quelqu'un jusqu'au bout de lui-même, lui permettre de s'accomplir, de trouver son destin 23 ». Mais ces mots et expressions se dépasser, ultime, jusqu'au bout de..., etc., ne sont pas au-dessus de tout soupçon. Ne font-ils pas écho à l'idéologie mortifère de la performance, de l'excellence, de la qualité totale? Les redéfinitions contemporaines de la spiritualité sont curieusement en phase avec une culture qui a la passion du risque, de l'extrême, des nouvelles frontières, de la prouesse, de l'exploit. Il est légitime de demander si certaines spiritualités actuelles ne sont pas au service de la cohésion de sociétés qui, à travers des valorisations de la virtuosité et de la réussite à la Bill Gates, opèrent des clivages entre des sauvés et des damnés... Ou si ces spiritualités ne sont pas l'opium de tous ceux et celles que le destin condamne à la médiocrité... L' « éthique » de la performance appelle constam ment au dépassement de soi; cette exigence écrasante ne demande-telle pas dès lors l'invention de spiritualités qui puissent donner sens à l'épreuve et à l'exaltation d'aller vers les sommets ultimes? Celles-ci

23 M. LAGUË, "Nommer et s'approprier sa spiritualité », Église et Théologie 29 (1998), pp. 7 et 15. 
sont peut-être le «ciment» identitaire des gagnants et des perdants de nos sociétés $24 \ldots$

Ces questions invitent à demander de quelle vision de l'être humain les discours contemporains sur la spiritualité sont porteurs. On peut y craindre le danger que le sens et le but de l'existence ignorent la traversée des réalités humaines concrètes. Devant une transcendance qui est opposition au biologique, au mécanique, etc., on peut rappeler ces lignes de Lévinas : "Le mouvement de transcendance se distingue de la négativité par laquelle l'homme mécontent, refuse la condition où il est installé... La négativité est incapable de transcendance ${ }^{25}$. " On peut rappeler également de ce même penseur ces belles méditations sur le visage d'autrui. Le visage, dans toute sa densité charnelle, le visage qui me regarde, dans sa résistance à l'objectivation, à la thématisation, est la transcendance. La philosophie de Lévinas convoque à interroger les concepts de la spiritualité qui font l'économie de la question de l'altérité. Car à y regarder un tant soit peu de près, il est remarquable que celle-ci soit absente bien souvent des promesses de réalisation ou d'accomplissement de soi. La transcendance définie dans les discours actuels est l'action d'un sujet, mouvement d'extase hors de soi; elle n'est pas le "chiffre" d'un autre que soi. Reconnaître une altérité n'est pas appel au surpassement, mais l'aveu de l'impossible maîtrise, l'humble aveu d'être dépassé. Autrui convoque à la réponse : c'est-à-dire à être dépassé par un regard qui ne peut laisser indifférent et à s'en tenir responsable.

24 Ces questions paraîtront peut-être blasphématoires à d'aucuns. Je les soumets ayant en mémoire que les églises chrétiennes ont souvent cautionné «spirituellement " des puissants et des gagnants : encore tout récemment, l'apartheid était un dogme dans certaines églises sudafricaines. La vigilance est de mise pour tous; la spiritualité du dépassement ne doit pas faire oublier les mots de Bill Gates: "You have the lunch or you are the lunch. *

25 E. LÉVINAS, Totalité et infini. Essai sur l'extériorité, Paris, Le Livre de Poche (Biblio - essais 4120), 1998, pp. 30.31. 


\section{La transcendance «en deçà " du spirituel et du religieux}

L'étroitesse reprochée à la religion peut être appliquée aussi aux réflexions sur la spiritualité. Le risque d'enfermement y est aussi présent. L'intérêt pour le spirituel aujourd'hui s'accompagne d'une critique légitime de la religion; l'étiquette système de dogmes, de rites, de normes et de pratiques est trop souvent justifiée. La parole d'un Autre adressée à un sujet est trop souvent étouffée, trahie par un "corps organisé de croyances". La méfiance à l'égard du religieux, méfiance qui n'est pas nouvelle, comme il a été dit, exige de celui-ci une incessante et difficile autocritique. Et celle-ci n'est possible que dans la liberté; elle en est même le signe le plus authentique. L'autocritique est le fruit du dialogue, du passage à travers la parole de l'autre, ce que les institutions religieuses tolèrent très mal. Une religion qui n'est pas travaillée par le dialogue avec la société, la culture, l'existence des humains est vouée à une mort prochaine. La désaffection à l'égard du christianisme en Occident semble bien en être l'illustration.

Mais les recherches spirituelles vagabondes de nos contemporains doivent aussi être mises à l'épreuve de l'autocritique. On sait que ces recherches insistent beaucoup sur l'importance de la conscience et sur la mise en œuvre jusqu'à l'extrême des pouvoirs humains ${ }^{26}$. En cautionnant les standards de réussite et d'excel lence, elles " enferment " à leur tour les hommes et les femmes dans des destins mortifères. Lévinas a " cherché en dehors de la conscience et du pouvoir, une notion d'être fondant la transcendance. " Et «la relation recherchée [lui] sembla inscrite dans la relation érotique. » Inscription surprenante! Mais Lévinas constate que "par la sexualité le sujet entre en rapport avec ce qui est absolument autre - ... - avec ce qui demeure autre dans la relation sans jamais se convertir en "mien" 27». À travers ces mots, il nous est indiqué encore que les cheminements spirituels ne peuvent ignorer que toute chair est

26 Et même sur le pouvoir "illimité » de la conscience, du subconscient, etc. N'est-ce pas là une participation "inconsciente" au refoulement de la mort, de la finitude en nos sociétés?

27 E. LÉVINAS, Totalité et infini, pp. 308-309. 
épiphanie d'un autre; que dans l'intense proximité à l'autre, une transcendance s'atteste.

Ces réflexions de Lévinas étonnent. La religion chrétienne a beaucoup parlé d'oubli et d'abnégation de soi - et la sexualité a été souvent le lieu où il fallait pratiquer cet oubli. Les nouvelles spiritualités lui opposent maintenant la réalisation de soi. Mais le terme transcendance n'enseigne-t-il pas que le soi n'est pas le dernier mot ${ }^{28}$ ? Que la transcendance commence ou donne naissance à la liberté quand celle-ci refuse toutes les réductions qu'elles soient religieuses ou spirituelles? Le texte biblique esquisse à divers endroits une transcendance qui survient dans le rapport à autrui. Comme JeanPierre Jossua le note avec beaucoup de sensibilité, il y est question d'un Dieu qui « juge le monde selon la justice qui y est faite aux pauvres ce qui oriente vers une certaine sorte de transcendance ${ }^{29}$. $\gg$ Il ne s'agit pas d'une fuite hors du monde matériel, biologique, psychologique, etc., ni d'un surpassement des limites pour aller jusqu'au bout de soi, mais d'un renversement de ce qui interdit la liberté à autrui. Cette "sorte" de transcendance est l'aune avec laquelle le religieux et le spirituel peuvent être critiqués. Déjà, le prophète Michée le suggérait: «Avec quoi me présenter devant le Seigneur, m'incliner devant le Dieu de là-haut? Me présenterai-je devant lui avec des holocaustes? Avec des veaux d'un an? Le Seigneur voudrait-il des milliers de béliers? des quantités de torrents d'huile? Donnerai-je mon premierné pour le prix de ma révolte? Et l'enfant de ma chair pour mon propre péché? On t'a fait connaître, ô homme, ce qui est bien, ce que le Seigneur exige de toi : Rien d'autre que le respect du droit, l'amour de la fidélité, la vigilance dans ta marche avec Dieu. » $(6,6-8)$ On ne peut imaginer plus vive critique de la religion que ces mots du prophète. Ils stigmatisent également les spiritualités lorsqu'elles proposent des harmonies qui occultent les souffrances des humains.

28 Voir E. LÉVINAS, Totalité et infini, p. 306: «Dans la conception classique, l'idée de la transcendance se contredit. Le sujet s'emporte dans sa transcendance." "

29 J.-P. JOSSUA, Le Dieu de la foi chrétienne, p. 13, souligné dans le texte. Et le texte se continue ainsi, en écho à la relation érotique proposée par Lévinas "qu'il [Dieu] passe au doigt de son peuple une alliance pour toujours". 
Michée indique les balises d'un chemin où le spirituel et le religieux peuvent attester de leur dif-férence, de ce qu'ils "portent dans leur ventre ".

La spiritualité rappelle avec justesse qu'il en va de la dignité de l'humain de ne pas l'enfermer dans le biologique, le mécanique, le système, les normes et les pratiques. Mais le religieux lui rétorque avec tout autant de justesse que la rencontre de l'humain, de son visage exige avec "vigilance " les médiations du langage, du droit, du symbole, des normes, etc., pour que cet humain soit accueilli jusque dans les blessures de sa chair. La spiritualité porte en elle le refus de l'enfermement; la religion porte les mots pour dire et vivre ce refus. L'une et l'autre peuvent encore se conjuguer pour qu'adviennent le sens et le but de l'existence. 